\title{
High energy resolution inorganic scintillators read by SiPM arrays
}

\author{
Miguel Garcia-Diez, Member, IEEE, Victor Sanchez-Tembleque, Luis Mario Fraile, Member, IEEE, Jose Manuel \\ Udias, Member, IEEE
}

\begin{abstract}
The high energy resolution inorganic scintillators as $\mathrm{LaBr}_{3}(\mathrm{Ce})$ have a great importance in the field of nuclear physics and medical applications since they offer great resolution in energy and time. On the other hand, SiPMs are gaining relevance in the fields mentioned above due to its advantages as compact size, invulnerability to magnetic fields, less voltage needed, etc. The goal of this work is to be able to use relatively large scintillators crystals $(25,4 \times 25,4 \mathrm{~mm}$ cylindrical), for that reason we can't use a single SiPM, because its size is much smaller than the size of the crystal, we use an array of $8 \times 8$ SiPMs of $3 \times 3 \mathbf{m m}^{2}$ in size from Ketek (model PA3325-WB-0808). With respect to the energy results we obtained the best value using the $\mathrm{LaBr}_{3}(\mathrm{Ce})(25,4 \times 25,4 \mathrm{~mm}$ cylindrical) coupled to the array, providing a resolution of 3,9\% (FWHM) for the $661 \mathrm{keV}$ photopeak.

For the timing results, we acquired measurements using Co-60 and Na-22 sources and the same $\mathrm{LaBr}_{3}(\mathrm{Ce})$ as scintillator crystal. Obtaining coincidence resolving times (FWHM) of 385 ps for Na22 and 337 ps for Co-60.
\end{abstract}

\section{INTRODUCTION}

$\mathrm{W}$ have evaluated the energy and time resolution of several scintillator crystals as LYSO, CeGAGG, CsI(Tl) and $\mathrm{LaBr}_{3}(\mathrm{Ce})$ coupled to a $8 \times 8 \mathrm{SiPMs}$ array from ketek (model PA3325-WB-0808). We obtained good energy resolution, specially with $\operatorname{LaBr}_{3}(\mathrm{Ce})$ scintillator crystal. The improvement of the energy resolution is due to the array surface size, which can cover the entire scintillator crystals.

When we used the scintillator crystal $\mathrm{LaBr}_{3}(\mathrm{Ce})$ coupled to a PMT (Hamamatsu R9779), the CRT (coincidence time resolution) that we obtained was 194 ps FWHM using Na-22 and 136 ps using Co-60 [1]. So our actual objetive is to achieve, at least, the same result with SiPMs for the same $\mathrm{LaBr}_{3}(\mathrm{Ce})$ crystal, with dimensions of $25,4 \times 25,4 \mathrm{~mm}$ (Cylindrical),

\section{EXPERIMENTAL SETUP}

The scintillators used in this work was an $\mathrm{CsI}(\mathrm{Tl})$ of

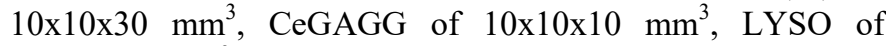
10x10x10 $\mathrm{mm}^{3}$ and a $\mathrm{LaBr}_{3}(\mathrm{Ce}) 25,4 \times 25,4 \mathrm{~mm}$ (Cylindrical) for the energy measurements, and two similar $\mathrm{LaBr}_{3}(\mathrm{Ce})$ of $25,4 \times 25,4 \mathrm{~mm}$ (Cylindrical) for the timing measurements.

Manuscript received December 16, 2019

Miguel Garcia Diez, Víctor Sánchez-Tembleque, Luis Mario Fraile and José Manuel Udías. from the Universidad Complutense de Madrid, Grupo de Física Nuclear, Facultad de Ciencias Físicas, Avda. Complutense s/n, E-28040 Madrid, Spain
All of the tests were done coupling the crystals to an array of 8x8 SiPMs from Ketek, model PA3325-WB-0808. The array is connected to a PCB, which add the anode of each single SiPM in parallel, then the signal goes to a transformer (model TC1-1-13MG2+), and we do the readout after of this transformer.

In the digital side, to digitalize the pulses we used the Domino Ring Sampling board (DRS4) developed at the Paul Sccherrer Institute (PSI) [2],[3]. The sampling speed used in the tests was $5 \mathrm{Gs} / \mathrm{s}$, storing the analog waveform in 1024 sampling cells per channel.

\section{MEASUREMENTS}

\section{A. Energy measurements}

For doing the measurements of energy we have used the scintillator crystals discussed above, the array of $8 \times 8$ SiPMs from Ketek an Eu-152, Cs-137, Na-22 and Co-60 as radioactive sources.

The measurement of the energy has been obtained doing a Simpson integral of the pulse after the whole pulse was digitalized. In this way we assign a value of energy to each pulse, and representing the pulses versus their energy value we obtain the energy spectra.

To measure the energy resolution of each photopeak we just need to adjust the photopeak to a Gaussian, and from the value of its FWHM we obtain the energy resolution.

TABLE I. RESULTS OF ENERGY RESOLUTION (\%,FWHM) FOR DIFFERENT PHOTOPEAKS USING VARIOUS CRYSTALS COUPLED TO AN ARRAY OF 8 X8 SIPMS

\begin{tabular}{lllll} 
Photopeak & $\mathrm{LaBr}_{3}(\mathrm{Ce})$ LYSO & CeGAGG & CsI(Tl) \\
\hline $340 \mathrm{keV}$ & 6,5 & 13,3 & 10,0 & 11,4 \\
$511 \mathrm{keV}$ & 4,5 & 11,2 & 8,0 & 9,9 \\
$662 \mathrm{keV}$ & 3,9 & 10,5 & 6,5 & 8,7 \\
$1170 \mathrm{keV}$ & 3,4 & 7,7 & 4,8 & 6,4 \\
$1275 \mathrm{keV}$ & 3,1 & 7,5 & 4,8 & 6,3 \\
$1330 \mathrm{keV}$ & 2,8 & 6,8 & 4,4 & 5,5
\end{tabular}




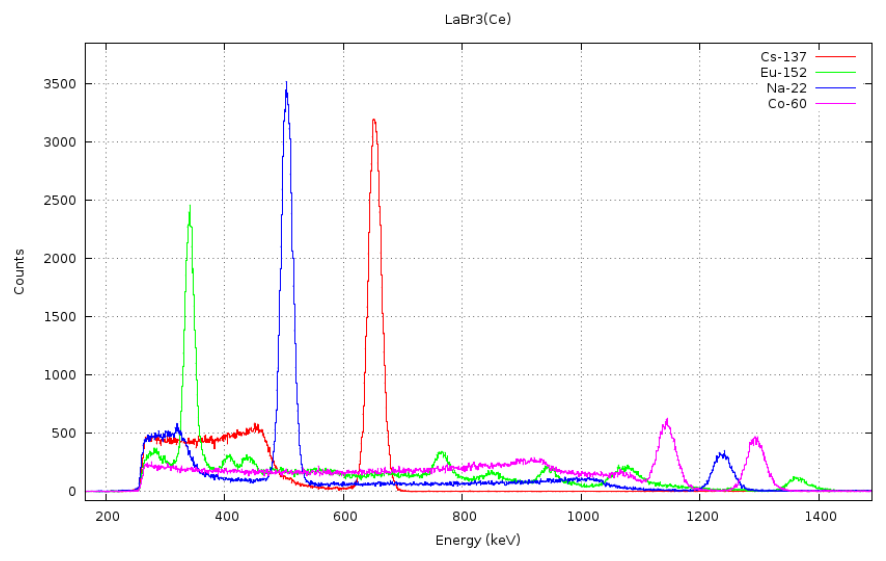

Fig. 1. Energy spectrum obtained using $\operatorname{LaBr}_{3}(\mathrm{Ce})$ coupled to the array of SiPMs using different radioactive sources.

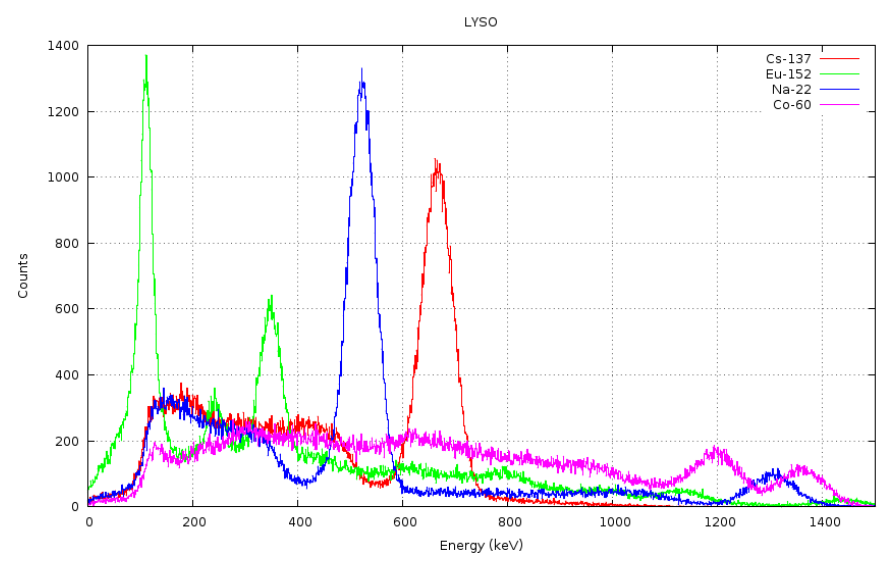

Fig. 2. Energy spectrum obtained using LYSO coupled to the array of SiPMs using different radioactive sources.

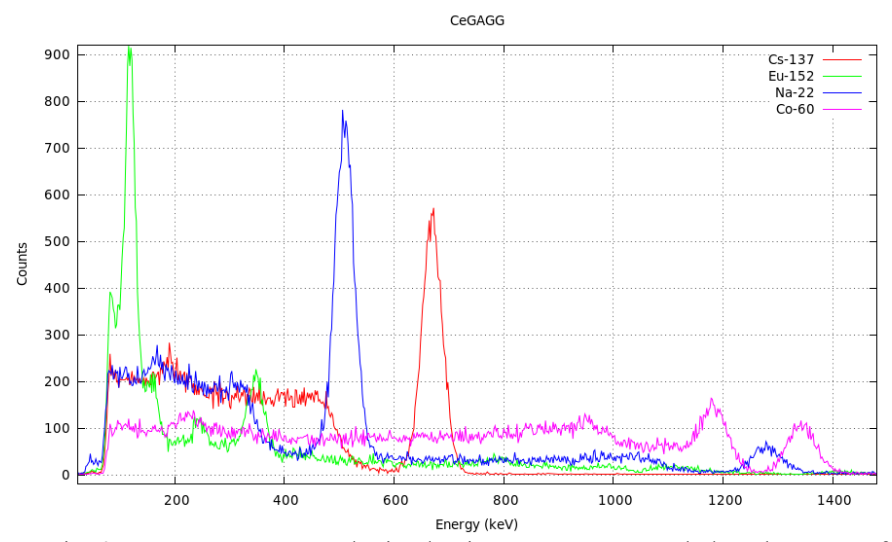

Fig. 3. Energy spectrum obtained using CeGAGG coupled to the array of SiPMs using different radioactive sources.

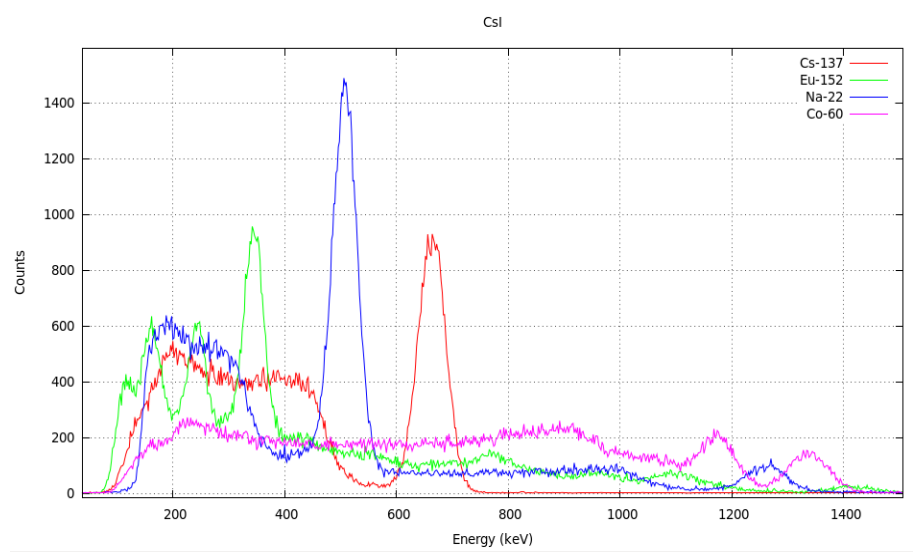

Fig. 4. Energy spectrum obtained using $\operatorname{CsI}(\mathrm{Tl})$ coupled to the array of SiPMs using different radioactive sources.

\section{B. Timing measurements}

For the timing measurement we used two similar $\operatorname{LaBr}_{3}(\mathrm{Ce})$ $(25,4 \times 25,4 \mathrm{~mm}$ Cylindrical) coupled to the two arrays of $8 \times 8$ SiPMs. As radioactive sources we used Na-22 and Co-60. The way of doing this test was, starting from the main signal, we divide in two, one for obtain the energy spectra and the other signal goes to a ZFL-500LN, an amplifier that will saturates the signal to provide us a ultra fast pulse for obtain the time resolution.

From the energy spectra we select de range of it that interests to us (the coincidence photopeak), in this way we will only work with the pulses that correspond to the coincidence events.

The time coincidence spectra was obtained using a leading edge algorithm [4], in which the trigger condition is set a threshold in the pulse at a percentage of its maximum. To optimize the value of this threshold we used a genetic algorithm [5].Then represent the time coincidence spectra and we obtain the temporal coincidence resolution from the FWHM.

TABLE II. TIME COINCIDENCE RESUlts (PS,FWHM) BETWEen LABR3(CE) COUPLED TO THE ARRAY OF 8X8 SIPMS.

\begin{tabular}{ll} 
Source & CRT \\
\hline Na-22 & 385 \\
Co-60 & 337
\end{tabular}




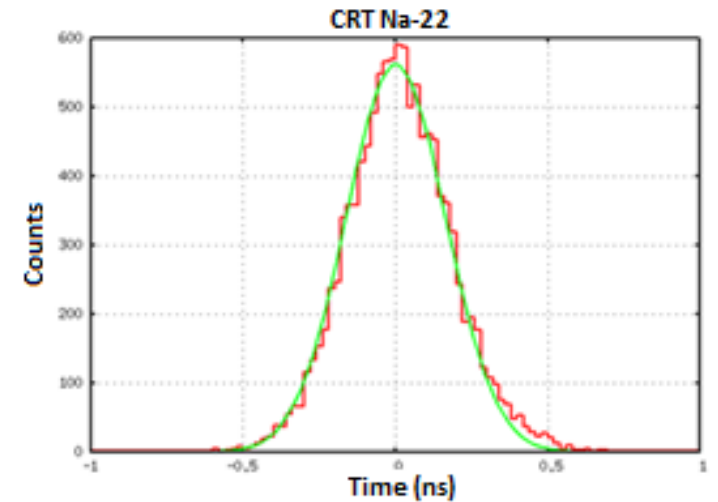

Fig. 5. Time coincidence spectra using $\mathrm{Na}-22$ as radioactive source

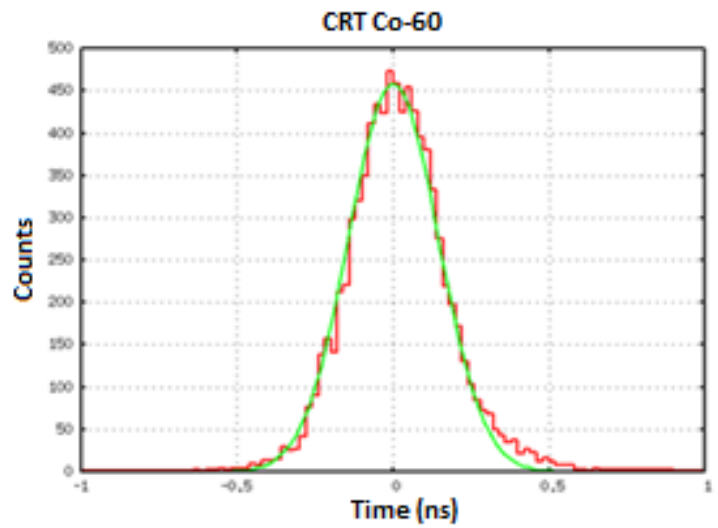

Fig. 6. Time coincidence spectra using Co-60 as radioactive source

\section{RESULTS}

The energy resolution results was better that the values that we obtained when we used a single $6 \times 6 \mathrm{~mm}^{2} \mathrm{SiPM}$, which are for the $661 \mathrm{keV}$ photopeak; $10,5 \%$ for LYSO, 6,5\% for CeGAGG, $8,5 \%$ for $\mathrm{CsI}(\mathrm{Tl})$ and the remarkable value of 3,9 $\%$ for $\mathrm{LaBr}_{3}(\mathrm{Ce})$

With respect to timing, after trying different combinations of amplifiers for make the pulse as faster as we can, the best result that we obtained was 385 ps when use the Na-22 source, and $337 \mathrm{ps}$ for the Co-60 source.

\section{CONCLUSIONS}

Energy and time resolution obtained with SiPM and large crystals has been limited due de difference in size between SiPM and crystals. In this test we verified that improving the surface of SiPMs using arrays we solve the problem. For that reason we obtain better energy and time resolution using an array against a single SiPM, so if we improve the electronics and thanks to the advantages that the new generations of SiPMS have, we can approach to the goal of having better CRT using SiPMs than PMTs.

\section{ACKNOWLEDGMENT}

This work have been supported by EU regional funds (RTC-2015-3772-1) Spanish MINECO through projects (FPA2015-65035-P, FPA2013-41267), CAM (S2013/MIT3024 TOPUS-CM) and by EU's H2020 under MediNet a Networking Activity of ENSAR-2 (grant agreement 654002)

\section{REFERENCES}

[1] V. Vedia, M. Carmona-Gallardo, L.M. Fraile, H. Mach, J. M. Udías, Performance evaluation of novel $\mathrm{LaBr} 3(\mathrm{Ce})$ scintillator geometries for fast-timing applications, Nucl. Instrum. Meth. 795 (2015) 144-150

[2] S. Ritt, "Design and performance of the $6 \mathrm{GHz}$ waveform digitizing chip DRS4,Nucl. Sci. Symp. Conf. Rec, Oct. 2008, pp. 1512-1515.

[3] S. Ritt, "DRS4 Handbook," Paul Scherrer Inst., Villigen, Switzerland, Rev. 0.9, 2008

[4] V. Sánchez-Tembleque, V. Vedia, L. M. Fraile, S. Ritt, J. M. Udías, Evaluation fot inogarnic scintillator for high performance ToF PET aplicacions, Nucl. Sci. Symp. Conf. Rec, Oct. 2015

[5] V. Sánchez-Tembleque, V. Vedia, L. M. Fraile, S. Ritt, J. M. Udías, New Algorithms for Improved Digital Pulse Arrival Timing With SubGsps ADCs, NIMA 2018 